Swarthmore College

Works

3-1-2003

\title{
X-Ray Spectral Diagnostics Of Neon Photoionization Experiments On The Z-Machine
}

David H. Cohen

Swarthmore College, dcohen1@swarthmore.edu

\section{J. J. MacFarlane}

J. E. Bailey

D. A. Liedahl

Follow this and additional works at: https://works.swarthmore.edu/fac-physics

Part of the Astrophysics and Astronomy Commons

Let us know how access to these works benefits you

\section{Recommended Citation}

David H. Cohen, J. J. MacFarlane, J. E. Bailey, and D. A. Liedahl. (2003). "X-Ray Spectral Diagnostics Of Neon Photoionization Experiments On The Z-Machine". Review Of Scientific Instruments. Volume 74, Issue 3. 1962-1965. DOI: 10.1063/1.1535261

https://works.swarthmore.edu/fac-physics/55

This work is brought to you for free by Swarthmore College Libraries' Works. It has been accepted for inclusion in Physics \& Astronomy Faculty Works by an authorized administrator of Works. For more information, please contact myworks@swarthmore.edu. 


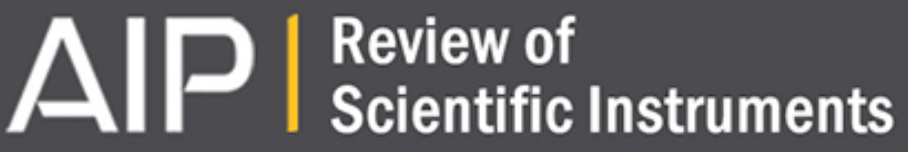

X-ray spectral diagnostics of neon photoionization experiments on the Z-machine

David H. Cohen, Joseph J. MacFarlane, James E. Bailey, and Duane A. Liedahl

Citation: Review of Scientific Instruments 74, 1962 (2003); doi: 10.1063/1.1535261

View online: http://dx.doi.org/10.1063/1.1535261

View Table of Contents: http://scitation.aip.org/content/aip/journal/rsi/74/3?ver=pdfcov

Published by the AIP Publishing

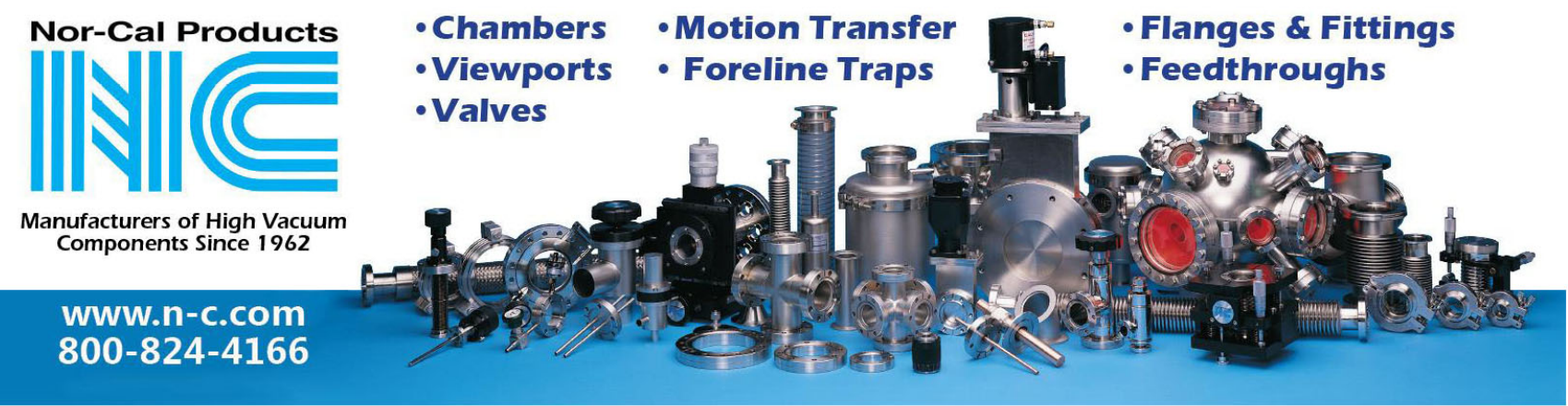




\title{
$X$-ray spectral diagnostics of neon photoionization experiments on the Z-machine
}

\author{
David H. Cohen \\ Department of Physics and Astronomy, Swarthmore College, 500 College Avenue, Swarthmore, \\ Pennsylvania 19081 and Prism Computational Sciences, 455 Science Drive, Suite 455, Madison, \\ Wisconsin 53711 \\ Joseph J. MacFarlane \\ Prism Computational Sciences, 455 Science Drive, Suite 455, Madison, Wisconsin 53711 \\ James E. Bailey \\ Sandia National Laboratory, P.O. Box 5800, Albuquerque, New Mexico 87185 \\ Duane A. Liedahl \\ Livermore National Laboratory, 7000 East Avenue, Livermore, California 94550
}

(Presented on 10 July 2002)

\begin{abstract}
We report on an initial spectroscopic study of low-density, x-ray photoionized neon with x-ray spectroscopy. These experiments, carried out on the Z-machine at Sandia, are optimized to produce a gradient-free, collisionless plasma, and to explore issues related to the rapid $\mathrm{x}$-ray photoionization of relatively cold, low-density plasmas. The initial experiments used time-integrated absorption spectroscopy, backlit by the pinch radiation, to determine the ionization balance in the gas cell. Future experiments will use time-resolved spectroscopy in both absorption and emission. The emission spectra are expected to be similar to those seen from photoionized astrophysical sources, such as x-ray binaries. Indeed, in addition to addressing basic plasma and atomic physics issues, these experiments are designed to help the astrophysical community better understand the new, high-resolution spectra being produced by the Chandra and XMM-Newton telescopes, and to benchmark spectral synthesis codes. () 2003 American Institute of Physics.
\end{abstract}

[DOI: $10.1063 / 1.1535261]$

With the launch of NASA's Chandra x-ray telescope ${ }^{1}$ in 1999, high-resolution X-ray spectroscopy of cosmic sources beyond the solar system became possible. This telescope and the similar XMM-Newton telescope, ${ }^{2}$ launched by the European Space Agency later that same year, comprise large (collecting areas of roughly $1 \mathrm{~m}^{2}$ ) nested hyperbolic and parabolic mirrors, instrument packages that include grating spectrometers (transmission gratings in the case of Chandra's HETGS and LETGS and reflection gratings in the case of XMM-Newton's RGS), and CCD detectors. These grating spectrometers span energies of $0.08 \mathrm{keV}$ to $10 \mathrm{keV}$ and have resolutions exceeding $\lambda / \Delta \lambda \sim 1000$ at some wavelengths. Throughputs of the gratings in combination with the mirror assemblies and CCD detectors are only in the tens of $\mathrm{cm}$. Future missions, primarily NASA's planned Constellation-X (Ref. 3) will have much larger throughput and potentially will achieve even higher spectral resolution using microcalorimeter arrays.

With the advent of high-resolution astrophysical spectroscopy, there is a new demand for detailed spectral models having an unprecedented degree of accuracy. Much attention over the past few decades has been paid to X-ray spectral modeling of collisional, or coronal, plasmas. However, many of the brightest $\mathrm{x}$-ray sources are not collisional but rather are dominated by photoionization. As shown by Liedahl et al., ${ }^{4}$ photoionized plasmas have a significantly different emission line spectrum than coronal plasmas having the same ionization distribution. Yet, the astronomical community has spent comparatively little effort benchmarking, testing, and analyzing spectral synthesis codes for photoionized plasmas. This will have to change, however, if we are to better understand some of the brightest and most interesting sources, including black hole and neutron star x-ray binaries and active galactic nuclei.

One important method for testing and benchmarking codes is to study $\mathrm{x}$-ray photoionized plasmas in the laboratory, where they can be controlled and characterized. If the ionization balance and temperature of an $\mathrm{x}$-ray photoionized plasma can be determined while its emission spectrum is observed, then the codes used to model astronomical x-ray sources can be tested with laboratory data, and the laboratory results can be used to guide the analysis of data from instruments like Chandra and XMM-Newton, and in the future, Constellation-X.

To this end, we have begun a series of ride-along shots on the Z-machine at Sandia to study the response of lowdensity gases to irradiance by strong $\mathrm{x}$ rays. The experimental packages consist of a cm-scale neon-filled cell with mylar windows, mounted several $\mathrm{cm}$ from the Z-machine's current return can, inside of which is the pinch itself-an imploding tungsten wire array, that can generate upwards of 200 TW of $\mathrm{x}$ rays in a $\sim 10 \mathrm{~ns}$ pulse, characterized by an emission temperature of roughly $200 \mathrm{eV}$.

Ultimately, we plan on making simultaneous, time- 
TOP VIEW
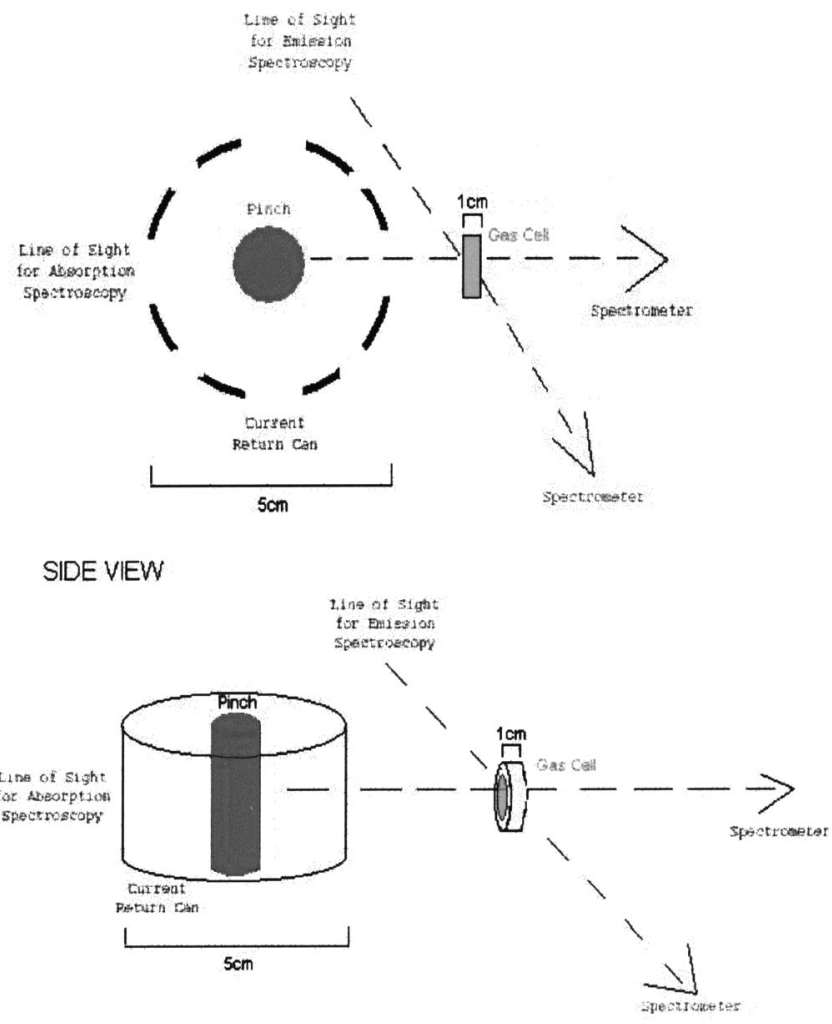

FIG. 1. Top and side views of the pinch, gas cell target, and diagnostic lines of sight. Note that the pinch both ionizes the plasma and acts as an absorption backlighter.

resolved absorption and emission spectroscopic measurements of the gas cell, which can be filled with a variety of gases, including mixtures. The absorption spectroscopy uses the pinch itself as a backlighter. See Fig. 1 for a schematic of the experiments. Experiments already completed have used 30 Torr neon $\left(n_{\text {atom }} \sim 10^{18} \mathrm{~cm}^{-3}\right)$ observed in absorption with a time-integrated spectrometer, and preliminary results have been reported by Bailey et al. ${ }^{5}$ We note that these experiments have relevance for basic atomic and radiation physics, as well as x-ray laser research, in addition to astrophysics.

Our interest is in the excitation/ionization kinematics and the physical conditions of the $\mathrm{x}$-ray photoionized plasma, and so we would like to minimize gradients, shocks, and collisional effects and isolate the atomic physics and spectral line formation physics. To that end, we begin with a low-density, uniform gaseous sample. We have performed experiments with the astrophysically relevant gas neon, and will eventually explore other gases, such as argon. A complementary set of experiments involving iron foils has also been performed on the Z-machine. ${ }^{6}$ The initial low density of the gas in our experiments minimizes hydrodynamic motion and plasma nonuniformities. However, the mylar walls, once heated and vaporized, will implode the gas in the cell. We have performed some initial scaling studies to explore how the thickness of the mylar walls can be varied to minimize compression and shock heating during the relevant phases of the experiment. See Fig. 2 for results of these calculations.

We have also performed simulations to explore the ef-
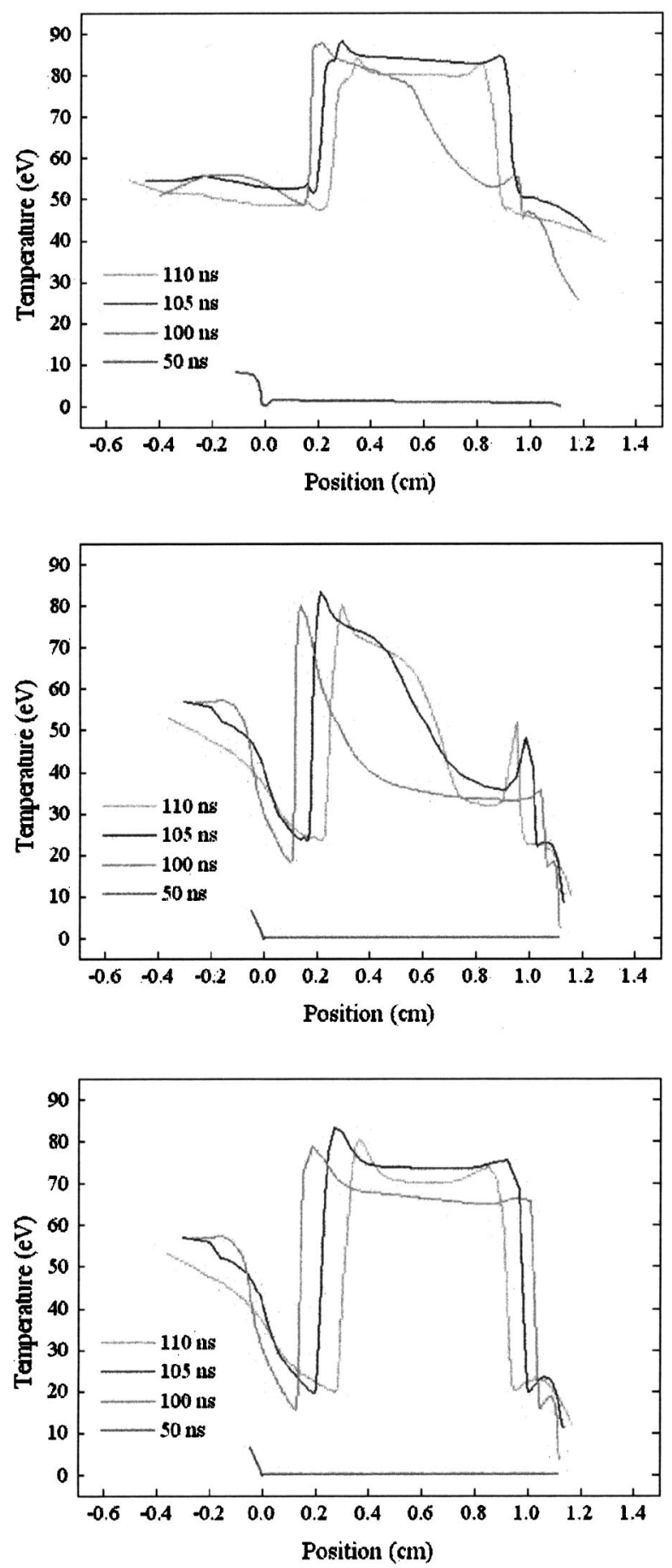

FIG. 2. Each panel displays four time snapshots $(50 \mathrm{~ns}, 100 \mathrm{~ns}, 105 \mathrm{~ns}$, and $110 \mathrm{~ns}$ ) of the plasma temperature in the cell for three different BUCKY simulations. The top simulation is for the experiments that have already been carried out, and assume 1.4 micron thick mylar walls and a gas fill density of $n_{\text {atom }}=10^{18} \mathrm{~cm}^{-3}$. The middle simulation has 5.6 micron walls and the same initial neon density. Note that the thicker walls put off the time when the mylar launches a shock wave into the gas. The peak drive power occurs between $100 \mathrm{~ns}$ and $105 \mathrm{~ns}$. And by $100 \mathrm{~ns}$, the wall-initiated shock has traveled less than $10 \%$ of the way into the gas in this second simulation. The lowest panel is from a simulation with a gas density of $n_{\text {atom }}$ $=10^{17} \mathrm{~cm}^{-3}$. Note that the gas is completely optically thin to the $\mathrm{x}$ rays at this density and thus the heating is more uniform in this case.

fects of gas fill density. Smaller densities are desirable to ensure that the plasma is photoionized and not collisional as well as to put the experiments closer to astrophysically relevant densities. But densities that are too small will not pro- 

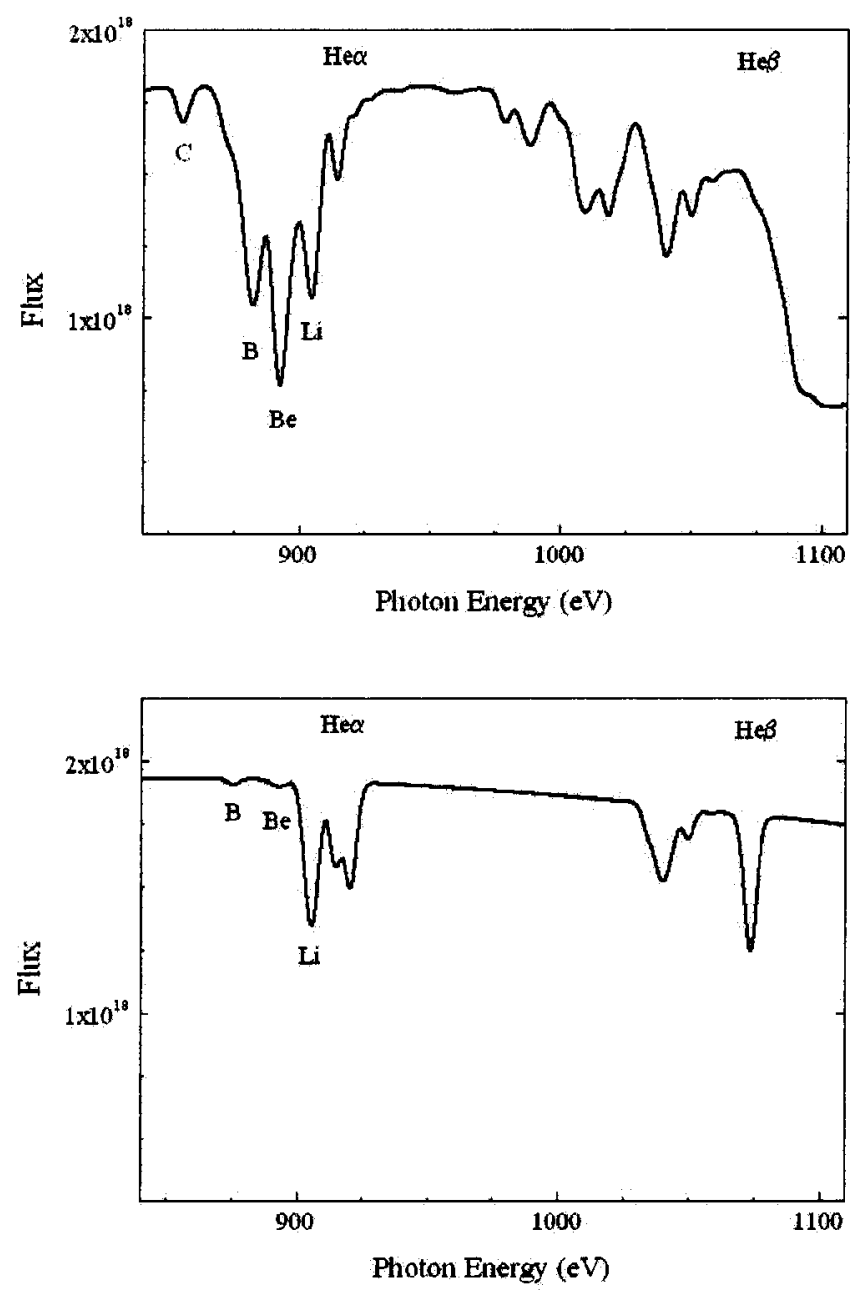

FIG. 3. SPECT3D simulations of backlit absorption spectra, for two different temperatures. Note how the ionization balance is readily inferred via the $K_{\alpha}$ absorption features, seen here at a resolution of $\lambda / \Delta \lambda \sim 300$. The corresponding $K_{\beta}$ features are seen at higher energies.

vide a strong enough absorption signal. Neon gas fill densities below $n_{\text {atom }}=10^{17} \mathrm{~cm}^{-3}$ (more than ten times less than the experiments we have already carried out) should optimize the tradeoff.

These hydrodynamic simulations are performed with the 1D Lagrangian code BUCKY. ${ }^{7}$ The input $\mathrm{x}$-ray field from the pinch is calculated using the VisRad (Ref. 8) viewfactor code. These calculations are also used to optimize the size and positioning of the gas cell. Combined VisRad and BUCKY simulations indicate that we should be able to obtain ionization parameters $(\xi=4 \pi I / n)$ in excess of 100 (in cgs units), which is the value seen in x-ray binaries.

We ultimately postprocess the hydrodynamics simulation output in order to synthesize observed spectra, using SPECT3D,${ }^{9}$ with atomic levels and transition rates calculated by ATBASE. ${ }^{10}$ In Fig. 3 we show a representative set of neon $K_{\alpha}$ absorption spectra, for different physical conditions in the gas cell. These calculations will be compared to timedependent absorption spectra backlit by the pinch itself, and will provide one constraint on the spectral modeling codes. We will also be observing time-resolved $\mathrm{x}$-ray emission spectra in future experiments. A representative emission line spectrum is shown in Fig. 4.
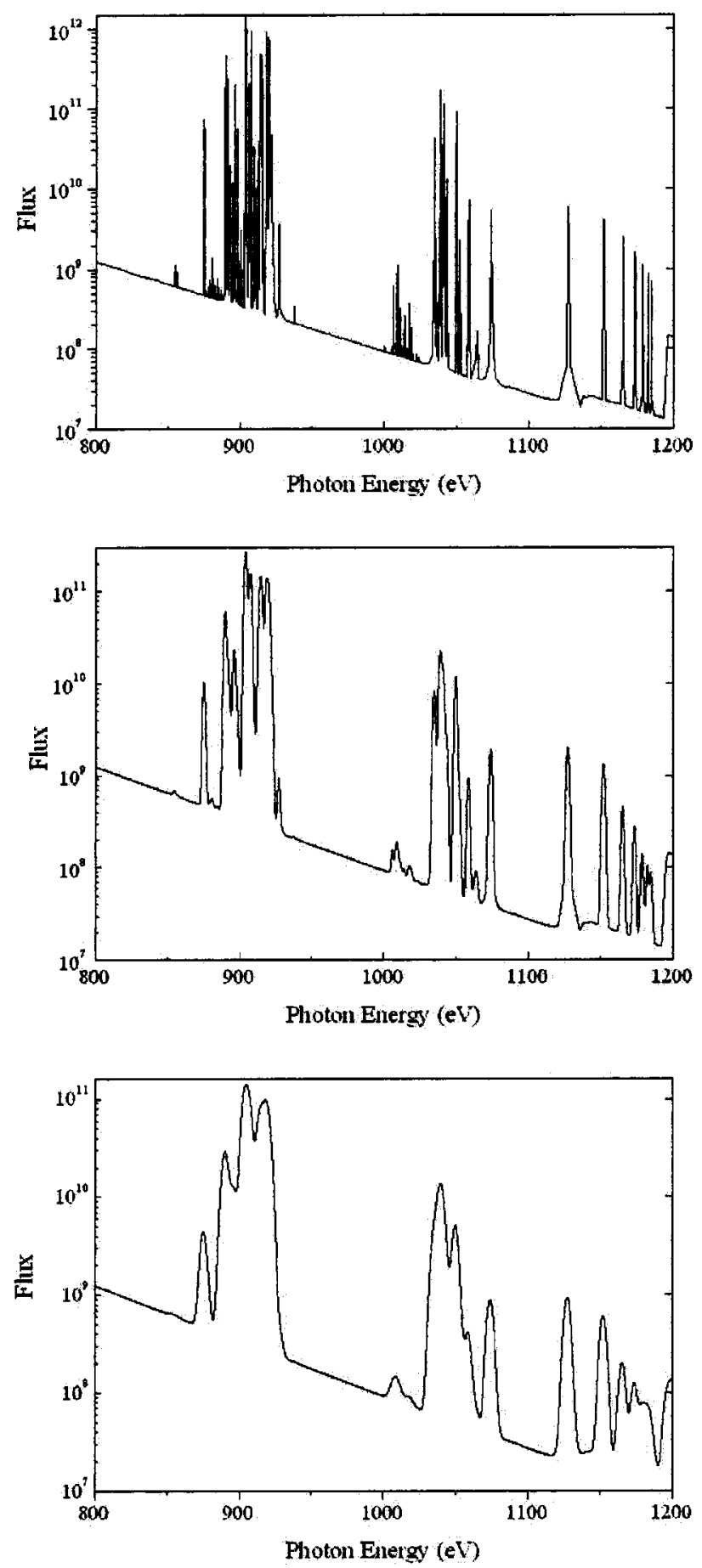

FIG. 4. A neon emission line spectrum synthesized with SPECT3D and based on conditions from the hydrodynamics simulations. The spectral resolution decreases moving down in this figure, from infinite on top to $\Delta E$ $=2 \mathrm{eV}$ in the middle panel and $\Delta E=5 \mathrm{eV}$ in the bottom panel. To test spectral codes in detail, we will need data with better spectral resolution than is seen in the lowest panel.

By producing a relatively gradient-free $\mathrm{x}$-ray photoionized plasma in the laboratory, and characterizing it while also measuring its emission spectrum, it will be possible to provide important information relevant to the astrophysical community using the Z-machine $\mathrm{x}$-ray source.

This research was made possible by Grant CC5489 from the Research Corporation. 
${ }^{1}$ Chandra Proposers Observatory Guide, http://cxc.harvard.edu/udocs/docs/ docs.html

${ }^{2}$ XMM-Newton Users' Handbook, http://xmm.vilspa.esa.es/external/ xmm_user_support/documentation/uhb_frame.shtml

${ }^{3}$ Constellation-X Mission Design, http://constellation.gsfc.nasa.gov/docs/ design/design_summary.html

${ }^{4}$ D. A. Liedahl, S. M. Kahn, A. L. Osterheld, and W. H. Goldstein, Astrophys. J. 350, L37 (1990).

${ }^{5}$ J. E. Bailey, D. H. Cohen, G. A. Chandler, M. E. Cuneo, M. E. Foord, R. F. Heeter, D. Jobe, P. Lake, D. A. Liedahl, J. J. MacFarlane, T. J. Nash, D. Nielson, R. Smelser, and W. A. Stygar, J. Quant. Spectrosc. Radiat. Transf. 71, 157 (2001).
${ }^{6}$ R. F. Heeter, J. E. Bailey, M. E. Cuneo, J. Emig, M. E. Foord, P. T. Springer, and R. S. Thoe, Rev. Sci. Instrum. 72, 1224 (2001).

${ }^{7}$ J. J. MacFarlane, G. A. Moses, and R. R. Petersen, University of Wisconsin Report No. UWFDM-984, 1 (1995).

${ }^{8}$ J. J. MacFarlane, A. R. Thomas-Cramer, P. Zeng, K. A. Park, M. W. Kissick, P. Woodruff, and I. E. Golovkin, Prism Computational Sciences Report No. PCS-R-037, 1 (2002).

${ }^{9}$ J. J. MacFarlane, Prism Computational Sciences Report No. PCS-R-039, 1 (2002).

${ }^{10}$ P. Wang, J. J. MacFarlane, and G. A. Moses, Phys. Rev. E 48, 3934 (1993). 